\title{
Evaluation de la productivité et de la mortalité de la poule locale sur le plateau du Sankaran, Faranah, G uinée, en 1993-1994
}

\author{
M. Mourad ${ }^{1}$ A.S. Bah ${ }^{1}$ G. Gbanamou ${ }^{1}$
}

\begin{abstract}
Mots-clés
Poulet - Poussin - Productivité - Elevage extensif - Mortalité - Maladie de Newcastle - Pullorose - Méthode statistique - Variation saisonnière Aviculture - Guinée.
\end{abstract}

\begin{abstract}
Résumé
Cet article traite de la productivité et de la mortalité de la poule de race locale en milieu villageois (plateau du Sankaran). La productivité de cette poule était très modeste, caractérisée par le nombre d'œufs par couvée $(10,05 \pm 0,15)$ et le nombre de couvées par an $(3,8 \pm 0,07)$. Le taux d'éclosion des œufs a varié de 42 à 100 p. 100. Le poids moyen des œufs était de 30,7 $\pm 0,03 \mathrm{~g}$. Le poids moyen des poussins éclos était de $22,8 \pm 0,23 \mathrm{~g}$. Le taux de fertilité s'est élevé à 87,5 p. 100 et le taux de viabilité des poussins d'un jour était de 89,31 p. 100. La maladie de Newcastle et la typhose pullorose étaient les principales causes de mortalité chez les poules et les poussins (plus de 60 p. 100 des pertes). Le taux de mortalité brut annuel s'est élevé à 80 p. 100 chez les poules et à 73,16 p. 100 chez les poussins. Le taux de mortalité brut saisonnier s'est élevé notamment pendant les saisons humide et froide. L'effet de la saison sur la mortalité des poules et des poussins observé au moyen du test du chi $^{2}$ était très significatif $(P<0,005)$. Les coefficients de corrélation et la détermination entre ces deux variables n'étaient pas significatifs $(P>0,05)$ et étaient respectivement de $-0,639$ et 0,4081 . L'intervalle de confiance du coefficient de corrélation était de $-0,639 \pm 1,7384$. L'effet des saisons sur le taux d'éclosion n'était pas significatif $(P>0,05)$. Les effets des saisons, des maladies et leur interaction étaient très significatifs $(P<0,005)$ sur le taux de mortalité des poussins. Le sex-ratio coq/poule était de 38 p. 100. Les éleveurs ont gardé 28 p. 100 des volailles pour le renouvellement du cheptel.
\end{abstract}

En Guinée, l'élevage avicole industriel n'est pas bien développé. Le marché de la volaille ne répond pas aux besoins alimentaires de la population. Cela est dû à l'insuffisance d'intensification de l'élevage des volailles. La plupart des poulets et des œufs vendus sur les marchés ruraux proviennent d'élevages villageois.

L'IEMVT-CIRAD * (5) a évalué la productivité de la poule locale africaine au Mali, au Burkina Faso et au Niger. Ces études ont

1. Centre de Recherche Zootechnique de Faranah (CRZF/IRAG), BP 1523, Conakry, Guinée

Tél : 411062 et 444262 ; fax : 415758 ou 4110

* CIRAD-EMVT depuis 1992 montré que la productivité de cette poule est faible (40 à 50 œufs par an, 3 couvées par an, un taux d'éclosion voisin de 60 p. 100 en hivernage et 50 p. 100 en saison sèche, un poids des poussins de 15 à $30 \mathrm{~g}$ à l'éclosion et un sex-ratio coq/poule inférieur à 10).

Une étude faite par Mcardle (6) en Inde a montré que la production de la race locale Desi en milieu villageois dans des conditions d'élevage extensif est d'environ 40 œufs par an. Cet auteur affirme que dans les conditions rurales, la seule amélioration logique et possible, particulièrement dans les zones éloignées, est celle permise, à court terme, par l'introduction de géniteurs améliorés. C'est une opération importante qui peut augmenter la productivité d'environ 50 p. 100 dans les mêmes conditions.

Au Mali et en Côte d'Ivoire, une étude de la production des races locales indique que le poids moyen des œufs dans les conditions naturelles est de $35 \mathrm{~g}$, le nombre d'œufs par an varie de 60 à 80 et le début de la ponte de 4 à 5 mois d'âge (7). 
En Guinée, les maladies constituent la principale contrainte de l'aviculture villageoise. D'après Courtecuisse et coll. (1), la principale maladie de la poule locale au Niger est la maladie de Newcastle qui cause 63 p. 100 de la mortalité.

\section{MATERIEL ET METHODES}

Entre septembre 1993 et août 1994, sur le plateau du Sankaran, quatre villages et 42 élevages ont été choisis au hasard. Tous les coqs et toutes les poules, respectivement 63 et 166, en âge de reproduction dans ces élevages ont été identifiés et suivis. L'unité zootechnique considérée comme la structure moyenne d'un troupeau se composait comme suit :

- poule reproductrice : 1

- coq adulte : 0,38

- œufs éclos : 31,5

- jeunes de 0 à 8 mois : 7,6

Des passages hebdomadaires et réguliers ont été effectués pour compter et peser les œufs par couvée, noter la date d'éclosion, calculer les taux d'éclosion, de viabilité et peser les poussins à l'âge d'un jour. L'autopsie de 193 poules et 152 poussins a été pratiquée au cours des passages afin d'identifier les principales causes de mortalité en milieu rural. En vue de confirmer l'examen clinique, des échantillons de foie, d'intestin et de rate ont été examinés pour déterminer les lésions pathognomoniques de chaque maladie.

Le système d'élevage pratiqué par tous les éleveurs était unique. Il n'y avait pas de poulailler utilisé comme abri mais par contre toutes les volailles étaient abritées dans les cases des propriétaires. Le système était de type extensif, les poules utilisaient les parcours aux alentours des cases. Quelques graines de céréales (maïs, brisures de riz, fonio...) leur étaient distribuées de temps en temps pour les habituer à la concession.

Le taux de mortalité brut annuel a été calculé pour les poussins et les poules en s'inspirant de la méthode utilisé par Faye et Pérochon (3) comme suit :

$\mathrm{Tm}=\mathrm{M} / \mathrm{E} \times 100$

où :

$\mathrm{M}=$ nombre de morts dans la période d'étude ;

$\mathrm{E}=$ effectif moyen de la population au cours de cette même période.

Au cours de cette étude, cinq périodes de l'année ont été observées :

- saison sèche froide (SSF) : décembre, janvier et février ;

- saison sèche chaude (SSC) : mars et avril ;

- début de saison humide (DSH) : mai et juin ;

- saison humide (SH) : juillet, août et septembre ;

- début de saison froide (DSF) : octobre et novembre.

Le taux de mortalité brut saisonnier pour les poules et les poussins est égal à : Ms/Es x 100

où :

Ms = nombre de cas de mortalité au cours d'une saison ;

Es $=$ effectif moyen des volailles présentes pendant cette saison.
Au cours de l'enquête, les pertes de volailles après un incident, comme une morsure de chien ou de chat, la capture par un rapace, ont été déclarées par les éleveurs. Ces pertes ont été groupées sous les termes de «mauvaise gestion ». La destination des produits avicoles des élevages suivis a été étudiée.

\section{Analyse statistique}

Les données concernant les taux de mortalité bruts saisonniers des poussins et des poules ont été comparées et testées en utilisant le test du chi $^{2}$ pour l'indépendance (Goulden, 4) afin de déterminer l'effet de la saison sur le taux de mortalité.

Les coefficients de corrélation et de détermination ont été calculés pour les variables taux de pluviométrie (X) et taux d'éclosion (Y) selon Snedecor et Cochran (9).

Les effets des facteurs saison, maladie et leur interaction ont été testés par analyse de variance pour le taux d'éclosion des poussins et le taux de mortalité des poules.

Dans le cas de la signification de F dans le tableau ANOVA, le test de Duncan (2) a été utilisé pour détecter la différence entre les moyennes.

\section{RESU LTATS}

Le tableau I montre les résultats des caractères de production et de reproduction de la poule locale. L'âge d'entrée en production était de $180 \pm 17$ jours, le nombre d'œufs par couvée était de 10,05 $\pm 0,15$, le nombre de couvées par an était de $3,78 \pm 0,07$, le taux d'éclosion était de 42 à 100 p. 100, avec une moyenne de 83,0 \pm 1 p. 100 , la moyenne du poids des œufs était de $30,74 \pm 0,03 \mathrm{~g}$, la moyenne du poids des poussins éclos était de $22,82 \mathrm{~g}$, le taux de fertilité était de 87,49 p. 100 et le taux de viabilité des poussins à un jour était de 89,31 p. 100.

Les résultats d'examen d'organes ont montré des lésions pathognomoniques :

- dans le cas de la maladie de Newcastle, des lésions hémorragiques sur les muqueuses du proventricule et du cloaque ont été identifiées :

- la variole s'est caractérisée sur le plan clinique par l'apparition de lésions pustuleuses et croûteuses au niveau de la peau (épithélioma contagieux). Elle était fréquente en saison humide, surtout chez les jeunes de 2 à 4 mois ;

\section{Tableau I}

Caractères de production et de reproduction de la poule locale, Faranah, Guinée, 1993-1994

\begin{tabular}{lc} 
Caractères & $\begin{array}{c}\text { Moyenne } \\
\text { 土 erreur standard }\end{array}$ \\
\hline Age d'entrée en reproduction & $180 \pm 17$ \\
Nombre d'œufs par couvée & $10,05 \pm 0,15$ \\
Nombre de couvées par an & $3,78 \pm 0,07$ \\
Taux d'éclosion (\%) & $83,00 \pm 1,00$ \\
Poids des œufs (g) & $30,74 \pm 0,03$ \\
Poids des poussins à l'éclosion (g) & $22,82 \pm 0,23$ \\
Taux de fertilité & 87,49 \\
Taux de viabilité des poussins & 89,31
\end{tabular}


- la typhose pullorose s'est caractérisée par : 1) un foie hypertrophié avec des bandes rougeâtres et jaunâtres, de couleur bronzée très caractéristique ; 2) une rate congestionnée et hypertrophiée de couleur violacée ou rouge brune ; 3 ) une entérite aiguë dans les intestins avec une vive inflammation située en particulier au niveau du duodénum ; 4) dans l'appareil génital, au niveau de l'ovaire en particulier, une inflammation avec des jaunes altérés et quelquefois rupturés chez les pondeuses.

Le tableau II décrit les causes de mortalité des poules adultes. La principale cause de mortalité de la poule en milieu villageois était la maladie de Newcastle (54,70 p. 100), suivie de la typhose $(26,91$ p. 100 , et de la variole $(10,99)$. La mauvaise gestion était responsable de 7,40 p. 100 de la mortalité.

Le tableau III décrit les causes de mortalité des poussins. La typhose pullorose était la principale cause de mortalité des poussins en milieu villageois (35,34 p. 100). La maladie de Newcastle et la variole y ont contribué respectivement à 25,31 et 18,17 p. 100. La mauvaise gestion était responsable de 21,18 p. 100 des pertes. Le taux de mortalité brut annuel des poussins s'est élevé à 73,16 p. 100. Le taux brut saisonnier était plus marqué en saisons humide et sèche froide (respectivement 26,31 et 22,67 p. 100).

Les tableaux IV et $\mathrm{V}$ décrivent les résultats du test du chi ${ }^{2}$ de l'indépendance chez les poules et les poussins afin de montrer l'effet des saisons de l'année sur le taux de mortalité. Les valeurs de chi ${ }^{2}$

\section{Tableau II}

Causes de mortalité des poules adultes,

Faranah, Guinée,

septembre 1993 - août 1994

\begin{tabular}{lcc} 
Causes & $\begin{array}{c}\text { Nb. de cas } \\
\text { de mortalité }\end{array}$ & $\%$ \\
\hline Maladie de Newcastle & 488 & 54,70 \\
Typhose pullorose & 240 & 26,91 \\
Variole & 98 & 10,99 \\
$\begin{array}{l}\text { Mauvaise gestion } \\
\text { et autre }\end{array}$ & 66 & 7,40 \\
\hline
\end{tabular}

sont toutes les deux très significatives $(\mathrm{P}<0,005)$ et respectivement égales à 86,84 , avec $\mathrm{ddl}=4$, et 36,18 , avec $\mathrm{ddl}=4$, chez les poules et les poussins. Ces résultats montrent l'incidence des saisons sur la mortalité des poules et des poussins (figures 1 et 2).

Le coefficient de corrélation (rho $=-0,6390$ ) entre la pluviométrie $(\mathrm{mm} / \mathrm{j})$ et le taux d'éclosion n'est pas significativement différent de $0(\mathrm{P}>0,05)$.

Le tableau VI montre les résultats de l'analyse de la variance du taux d'éclosion. Les saisons de l'année n'ont pas d'effet significatif $(\mathrm{P}>0,05)$ sur le taux d'éclosion malgré l'écart entre les taux par saison (figure 3). Cela montre que le taux d'éclosion dépend d'autres facteurs non compris dans le modèle statistique tels que le taux de fertilité des œufs, le logement, l'alimentation des poules... Cet écart peut quantitativement diminuer la productivité numérique des poussins du cheptel villageois.

Les moyennes des taux d'éclosion par saison sont dans le tableau VII. Le taux d'éclosion a baissé pendant les saisons sèche froide et humide $(0,80$ et 0,75$)$.

La figure 1 montre le taux de mortalité brut saisonnier des poules. La maladie de Newcastle était la principale maladie causant la mortalité en début de saison humide et en saison sèche froide. La typhose pullorose quant à elle se manifestait surtout en saison sèche chaude, en saison humide et au début de la saison froide.

Tableau IV

Nombres observés et escomptés de poules mortes et restantes

\begin{tabular}{|c|c|c|c|c|c|}
\hline \multirow[t]{2}{*}{ Saisons de l'année } & \multicolumn{2}{|c|}{ Mortes } & \multicolumn{2}{|c|}{ Restantes } & \multirow[t]{2}{*}{ Total } \\
\hline & 0 bservées & Escomptées & 0 bservées & Escomptées & \\
\hline Saison sèche froide & 329 & 249 & 1115 & 1195,6 & 1444 \\
\hline Saison sèche chaude & 115 & 192 & 1000 & 923 & 1115 \\
\hline Début de saison humide & 140 & 176 & 885 & 848 & 1025 \\
\hline Saison humide & 197 & 162 & 745 & 780 & 942 \\
\hline Début de saison froide & 111 & 113 & 548 & 546 & 659 \\
\hline Total & 892 & - & 4293 & - & 5185 \\
\hline $\mathrm{chi}^{2}$ & $86,84^{* * *}$ & & & & \\
\hline
\end{tabular}

*** très significatif $(\mathrm{P}<0,005)$

Causes de mortalité des poussins et des poulettes selon leur âge, Faranah, Guinée, septembre 1993 - août 1994

\begin{tabular}{lccc} 
Causes & $\begin{array}{c}\text { Nb.de cas } \\
\text { de mortalité }\end{array}$ & $\begin{array}{c}\text { Catégories } \\
\text { d'âge } \\
\text { au moment } \\
\text { de la mort } \\
\text { (mois) }\end{array}$ & $\%$ \\
\hline $\begin{array}{l}\text { Typhose pullorose } \\
\text { Maladie }\end{array}$ & 317 & $0-4$ & 35,34 \\
$\begin{array}{l}\text { de N ewcastle } \\
\text { Variole }\end{array}$ & 227 & $4-6$ & 25,31 \\
$\begin{array}{l}\text { Mauvaise gestion } \\
\text { et autre }\end{array}$ & 163 & $2-4$ & 18,17 \\
& 190 & $0-6$ & 21,18 \\
\end{tabular}




\section{Tableau V}

Nombres observés et escomptés de poussins morts et restants

\begin{tabular}{lccrrrr} 
Saisons de l'année & \multicolumn{2}{c}{ Morts } & \multicolumn{2}{c}{ Restants } & Total \\
\cline { 2 - 5 } & O bservés & Escomptés & O bservés & Escomptés \\
Saison sèche froide & 278 & 245 & 1226 & 1259 & 1504 \\
Saison sèche chaude & 131 & 176 & 155 & 948 & 904 & 1079 \\
Début de saison humide & 133 & 141 & 817 & 795 & 950 \\
Saison humide & 180 & 97 & 504 & 497 & 864 \\
Début de saison froide & 90 & - & 4179 & - & 594 \\
Total & 812 & & & & 4991 \\
chi ${ }^{2}$ & $36,18 * * *$ & & & &
\end{tabular}

$* * *$ très significatif $(\mathrm{P}<0,005)$

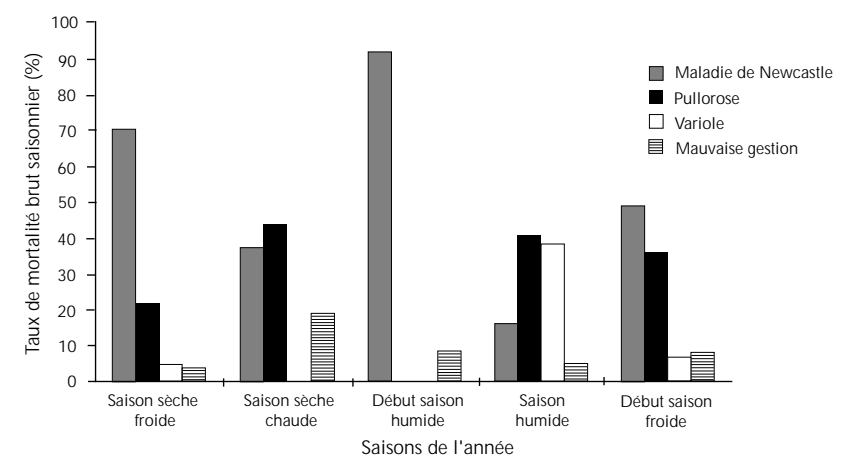

Figure 1 : taux de mortalité brut saisonnier des poules locales à Faranah, Guinée, septembre 1993 - août 1994.

\section{Tableau VI}

Analyse de la variance du taux d'éclosion (fonction saison)

\begin{tabular}{lrrccr}
$\begin{array}{l}\text { Sources de } \\
\text { variation }\end{array}$ & ddl & SCE & MCE & F \\
\hline $\begin{array}{l}\text { Entre saisons } \\
\text { Erreur }\end{array}$ & 4 & 0,13945527 & 0,0342632 & \\
& 169 & 2,92165795 & 0,0172879 & 2,0 \\
Total & 173 & 3,06111322 & & \\
\hline
\end{tabular}

La valeur $\mathrm{F}$ est non significative $(\mathrm{P}>0,05)$

Les résultats de l'analyse de la variance du taux de mortalité sont dans le tableau VIII. Les saisons de l'année et les maladies ont un effet très significatif $(\mathrm{P}<0,005)$ sur la mortalité des poussins. Les moyennes des taux de mortalité pour les deux facteurs étudiés (saisons de l'année et maladies) sont dans le tableau IX.

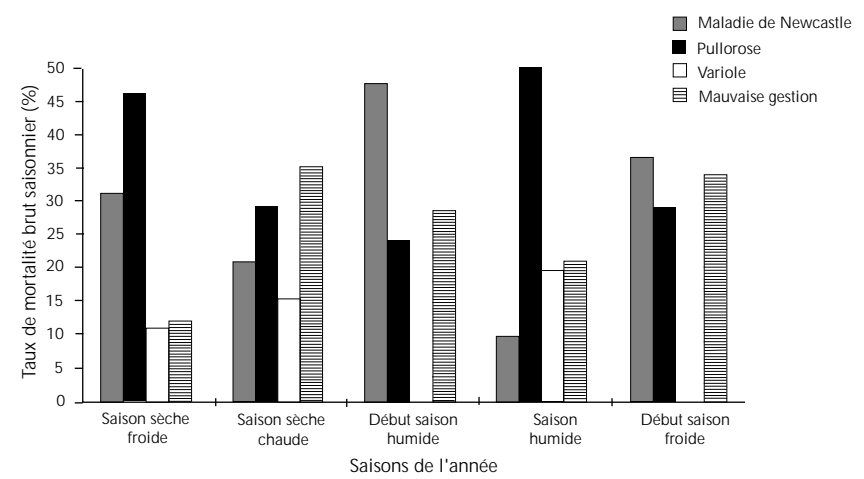

Figure 2 : taux de mortalité brut saisonnier des poussins dus aux différentes maladies à Faranah, Guinée, septembre 1993 - août 1994.

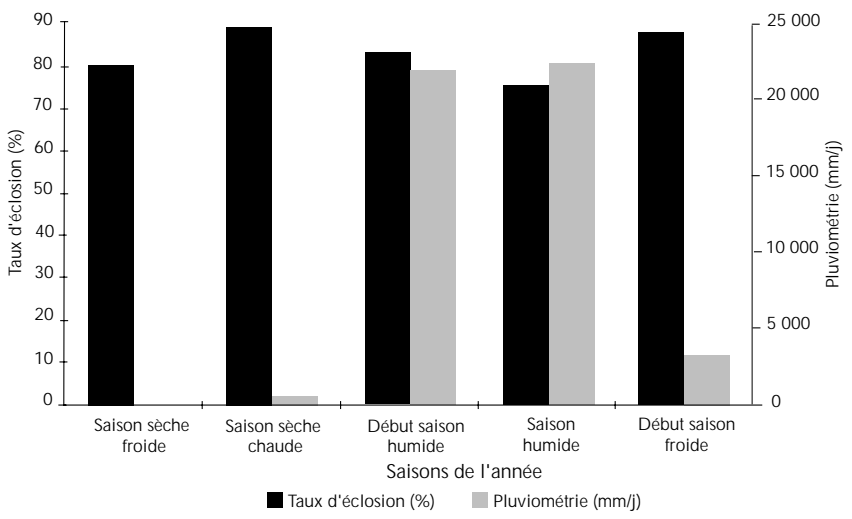

Figure 3 : taux d'éclosion et pluviométrie à travers les saisons de l'année.

L'interaction (saisons de l'année $\mathrm{x}$ maladies) était très significative et a révélé qu'une maladie entrainait une plus grande mortalité que les autres durant une saison donnée.

La figure 4 montre les résultats de la destination des produits avicoles au cours de la période d'étude. 
Tableau VII

Moyenne et erreur standard du taux d'éclosion

\begin{tabular}{lc} 
Saisons de l'année & $\begin{array}{c}\text { Moyenne } \\
\pm \text { erreur standard }\end{array}$ \\
\hline Saison sèche froide & $0,80 \pm 0,006$ \\
Saison sèche chaude & $0,89 \pm 0,004$ \\
Début de saison humide & $0,83 \pm 0,002$ \\
Saison humide & $0,75 \pm 0,005$ \\
Début de saison froide & $0,88 \pm 0,005$
\end{tabular}

Tableau IX

Moyenne et erreur standard du taux de mortalité selon les saisons et les maladies

\begin{tabular}{ll} 
Facteurs & $\begin{array}{c}\text { Moyenne } \\
\pm \text { erreur standard }\end{array}$ \\
\hline Saisons de l'année & \\
Saison sèche froide & $0,1776 \pm 0,0198^{\mathrm{g}}$ \\
Saison sèche chaude & $0,0884 \pm 0,0150^{\mathrm{f}}$ \\
Début de saison humide & $0,1737 \pm 0,0239^{\mathrm{g}}$ \\
Saison humide & $0,1798 \pm 0,0240^{\mathrm{g}}$ \\
Début de saison froide & $0,1719 \pm 0,0235^{\mathrm{g}}$ \\
Maladies & \\
Typhose pullorose & \\
Maladie de Newcastle & $0,1335 \pm 0,0146^{\mathrm{a}}$ \\
Variole & $0,1367 \pm 0,0164^{\mathrm{a}}$ \\
Mauvaise gestion & $0,1673 \pm 0,0281^{\mathrm{a}}$ \\
\end{tabular}

Les moyennes ayant les exposants a et b sont significativement différentes $(\mathrm{P}<0,05)$

Les moyennes ayant les exposants $\mathrm{f}$ et $\mathrm{g}$ sont significativement différentes $(\mathrm{P}<0,01)$

\section{DISCUSSION}

Cette étude montre que l'âge d'entrée en production des poules était de $180 \pm 7$ jours, résultat semblable à celui obtenu par Saunders (8) en milieu villageois, mais différent au niveau des référentiels généraux indiqués dans le Mémento de l'agronome (7), suite à une étude faite au Mali et en Côte d'Ivoire où l'âge d'entrée en ponte variait entre 120 et 150 jours. Cette différence peut être liée à la race exploitée et aux conditions du milieu dans ces deux pays. La poule locale produit 10,5 $\pm 0,15$ œufs par couvée avec un nombre de couvées égal à $3,8 \pm 0,07$ par an. Les résultats de cette étude sont à peu près semblables à ceux obtenus par l'IEMVT-CIRAD (5) qui a trouvé une production de trois couvées par an et de 15 œufs par couvée chez la poule locale au Mali, au Burkina Faso et au Niger, Saunders (8) une production de trois couvées par an et de 10 à 15 œufs par cycle de ponte en Haute Volta, et Mcardle (6) une production de 40 œufs par an en Inde.

Le taux d'éclosion de la poule locale était de 42 à 100 p. $100(83,0$ \pm 1 p. 100). Ce résultat est proche de celui obtenu par Saunders (8)

\section{Tableau VIII}

Analyse de la variance du taux de mortalité des poules (fonction saisons et maladies)

\begin{tabular}{lrcll}
$\begin{array}{l}\text { Sources de la } \\
\text { variation }\end{array}$ & ddI & SCE & MCE & F \\
\hline Entre saisons (S) & 4 & 0,674 & 0,1685 & $3,76^{* * *}$ \\
Entre maladies (M) & 3 & 0,671 & 0,223666 & $4,99 * * *$ \\
Interaction (S x M) & 12 & 1,3792 & 0,114933 & $2,57 * * *$ \\
Erreur & 498 & 22,2838 & 0,0447466 & \\
& 517 & 25,0 & - & \\
Total & & & &
\end{tabular}

*** très significatif $(\mathrm{P}<0,005)$
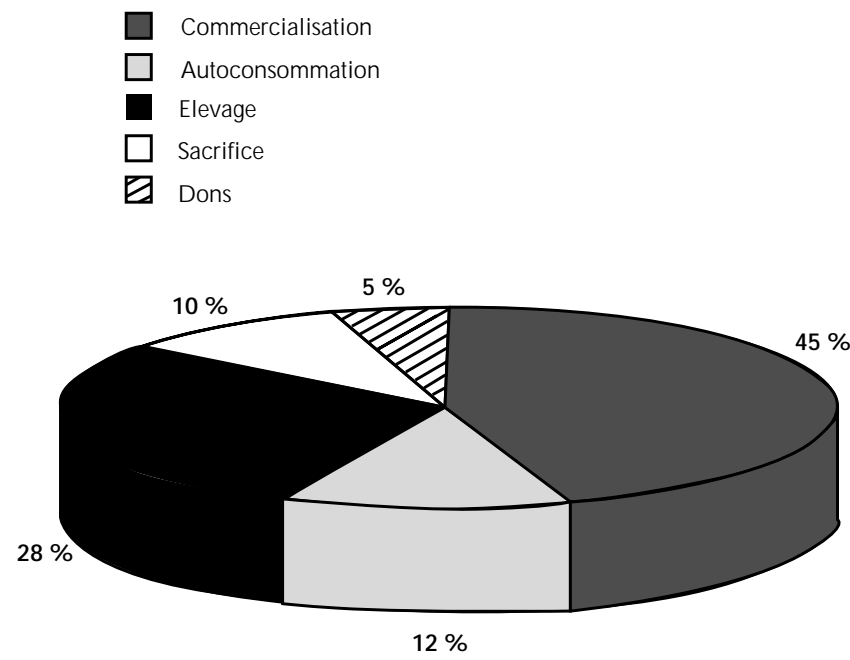

Figure 4 : destination des produits avicoles, Faranah, Guinée, 1993-1994.

et l'IEMVT-CIRAD (5) en hivernage ( 80 p. 100) et peut être interprété par la ressemblance du climat d'hivernage en pays sahéliens et la saison sèche en Guinée. Les mêmes auteurs ont ajouté que le taux d'éclosion décroît en dessous de 50 p. 100 en saison sèche.

Le poids moyen des œufs était de 30,74 $\pm 0,03 \mathrm{~g}$. Les résultats de cette étude sont inférieurs à ceux notés dans le Mémento de l'agronome (7) chez la poule locale en Côte d'Ivoire et au Mali (poids moyen des œufs égal à $35 \mathrm{~g}$ ). Cette différence peut être liée à la race exploitée et aux conditions d'élevage dans ces deux pays.

Le taux de fertilité était de 87,5 p. 100 , ce qui a semblé modeste aux auteurs, et le taux de viabilité des poussins à un jour était de 89,3 p. 100. Le poids moyen des poussins à l'éclosion était de 22,8 $\pm 0,23 \mathrm{~g}$. Ce résultat était semblable à la moyenne de $15-30 \mathrm{~g}$ obtenue par l'IEMVT-CIRAD (5) et comparable à celle de 21,6 g obtenue par Saunders en milieu villageois (8).

Le sex-ratio coq/poule était de 38 p. 100 en milieu rural, ce qui est très élevé par rapport à celui obtenu par l'IEMVT-CIRAD (5), inférieur à 10 p. 100. 
Le fait que les éleveurs aient un effectif élevé de coqs dans leur cheptel reflète bien la tradition villageoise de garder un bon nombre de coqs destinés aux dons, aux sacrifices et à la vente. Cette pratique diminue la productivité de la poule par manque de sélection raisonnée. Il est recommandé de maitriser le choix des caractères (chair et ponte), de contrôler les performances et d'appliquer la sélection de géniteurs par la pratique du chaponnage d'autres coqs destinés à l'abattage afin d'améliorer le caractère chair. Pour les poules, la sélection permet d'améliorer non seulement ces deux caractères, mais aussi ceux de la reproduction en milieu villageois.

D'un point de vue zootechnique, les auteurs reprennent l'affirmation de Mcardle (6) : dans des conditions rurales la seule amélioration logique et possible, en particulier dans les zones éloignées, est celle menée à court terme par le croisement des poules locales avec des géniteurs d'une race amélioratrice. Les auteurs recommandent aussi d'améliorer en parallèle les facteurs environnementaux (logement, ration équilibrée, soins vétérinaires et vaccinations). La cause principale de mortalité de la poule locale en milieu villageois était la maladie de Newcastle (54,70 p. 100), suivie de la typhose pullorose $(26,91$ p. 100$)$ et de la variole (10,99 p. 100). La mauvaise gestion était responsable de 7,40 p. 100 des cas de mortalité. Ces résultats sont très proches de ceux obtenus par l'IEMVT-CIRAD (5) dans les trois pays du Sahel où la maladie de Newcastle était la principale cause de mortalité. Elle peut décimer 80 à 100 p. 100 des troupeaux.

D'autres maladies, comme la variole, la typhose pullorose et le choléra aviaire, sont responsables de grandes pertes dues à l'absence de vaccination. Courtecuisse et coll. (1) ont trouvé, dans un échantillon de 230 poules, 47 p. 100 contaminées par la pullorose, contre 27 p. 100 dans cette étude. Pour un autre échantillon qui a été vacciné, 47 p. 100 des poules ont été trouvées contaminées par la maladie de Newcastle contre 54,7 p. 100 dans cette étude.

Le taux de mortalité brut annuel des poules s'est élevé à 80 p. 100 dans cette étude, résultat semblable à celui obtenu par l'IEMVTCIRAD (5). Dans une autre étude où la perte était plus importante, le taux de mortalité brut annuel a atteint 99 p. 100 la première année chez la poule locale en milieu villageois. La perte a été réduite de 84 p. 100 la deuxième année grâce aux soins vétérinaires et à la vaccination contre la maladie de Newcastle (Saunders, 8).

Les résultats du test $\mathrm{du} \mathrm{chi}^{2}$ chez les poules et chez les poussins montrent l'effet très significatif $(\mathrm{P}<0,005)$ des saisons sur le taux de mortalité (figures 1 et 2). La perte a eu lieu au cours des saisons humide et sèche froide.

La figure 4 montre la destination des produits avicoles au cours de la période d'étude. Les résultats obtenus dans les trois pays du sahel par l'IEMVT-CIRAD (5) montrent que dans ces pays l'autoconsommation et les volailles destinées au renouvellement du cheptel s'élèvent respectivement à 25 et 5 p. 100, tandis que dans cette étude, l'autoconsommation et les volailles destinées au renouvellement du cheptel étaient respectivement de 12 et 28 p. 100.

\section{- CONCLUSION}

La productivité de la poule locale était très faible au niveau de tous les caractères de production et de reproduction. Dans le système extensif étudié, les principales contraintes identifiées étaient d'ordre sanitaire et de gestion dans le milieu villageois. Les résultats de cette étude ont montré un effet non significatif $(\mathrm{P}>0,05)$ des saisons et de la pluviométrie sur le taux d'éclosion. Au plan national, cet effet peut causer une perte importante à l'éclosion.

Les effets des saisons, des maladies et de la gestion sur la mortalité des poussins étaient très significatifs $(\mathrm{P}<0,005)$.

Cela montre l'importance du logement, de la vaccination et des soins vétérinaires en milieu rural. Il est recommandé d'assurer les facteurs environnementaux (logement, santé, ration équilibrée et bonne conduite) avant l'application de l'amélioration génétique.

\section{Remerciements}

Les auteurs remercient M. Cécé Thea, CTE, pour sa participation aux travaux pratiques sur le terrain. Ils tiennent à remercier également tous les éleveurs pour leur coopération.

\section{BIBLIO G RAPHIE}

1. COURTECUISSE C., JAPIOT F., BLOCH N., DIALLO I., 1990. Enquêtes sérologiques sur les maladie de Newcastle et de Gumboro, la pasteurellose et la pullorose chez des poules de race locale au Niger. Revue Elev. Méd. vét. Pays trop., 43 : 27-29.

2. DUNCAN N.B., 1955. Multiple range and multiple $F$ test. Biometrics, $11: 1-24$.

3. FAYE B., PERO CHON L., 1995. La mortalité des vaches laitières dans I'enquête écopathologique - Bretagne. Vet. Res., 26 : 124-131.

4. GOULDEN C.H., 1952. Methods of statistical analysis, 2nd ed. NY, USA, John Wiley.

5. IEM VT-CIRAD, MINISTERE DE LA COOPERATION ET DU DEVELO PPEMENT, 1989. Développement de l'aviculture traditionnelle en Afrique tropicale. Maisons-Alfort, France, IEMVT-CIRAD, Paris, France, Ministère de la coopération et du développement. (Fiches techniques d'élevage tropical $n^{\circ} 2$ )

6. MCARDLE A.A., 1972. Méthodes de production avicole dans les zones en voie de développement. World Anim. Rev., (2) : 28.

7. MIN ISTERE DE LA COOPERATION ET DU DEVELO PPEMENT, 1991. M émento de I'agronome, $4^{\mathrm{e}}$ éd. Paris, France, Ministère de la coopération et du développement.

8. SAUNDERS M.J., 1984. Aviculture traditionnelle en Haute Volta. Synthèse des connaissances actuelles et réflexion autour d'une expérience de développement (1979-1984).

9. SNEDECOR G.W., COCHRAN W.G., 1976. Statistical methods. Ames, lowa, USA, lowa State U niversity Press.

Reçu le 13.6.96, accepté le 11.2.98 


\section{Summary}

Mourad M., Bah A.S., G banamou G. Productivity and mortality of local poultry in the plateau of Sankaran Faranah, Guinea, in 1993-1994

The productivity and mortality of the local poultry breed in a village environment in the plateau of Sankaran, Guinea, is described. Poultry productivity was very modest, characterized by the number of eggs per brood $(10.05 \pm 0.15)$ and broods per year $(3.8 \pm 0.7)$. Its hatching rate ranged from 42 to $100 \%$. The egg mean weight was $30.7 \pm 0.03 \mathrm{~g}$. The chick mean weight was $22.8 \pm 0.23 \mathrm{~g}$ at hatching. The fertility rate was $87.5 \%$ and chick viability rate was $89.31 \%$ at one day of age. Newcastle and typhosis pullorum diseases were the main causes of hen and chick mortality (more than $60 \%$ of losses). The annual raw mortality rates were 80 and $73.16 \%$ for hens and chicks, respectively. The seasonal raw mortality rate increased notably during the rainy and cold seasons. The chi ${ }^{2}$ test showed a highly significant effect $(P<0.005)$ of seasons on hen and chick mortality. Correlation and determination coefficients between these two variables, $-0,639$ and 0.4081 , respectively, were not significant $(P>0.05)$. The confidence interval of the correlation coefficient was $-0,639 \pm 1.7384$. The season effect on the hatching rate was not significant $(P>0.05)$, whereas the effects of seasons, diseases and their interaction were highly significant $(P<0.005)$ on the chick mortality rate. The sex-ratio cock/hen was $38 \%$. The breeders retained $28 \%$ of birds for flock replacement.

Key words: Chicken - Chick - Productivity - Extensive husbandry - Mortality - N ewcastle disease - Pullorum disease Statistical method - Seasonal variation - Aviculture - Guinea.

\section{Resumen}

Mourad M., Bah A.S., G banamou G. Evaluación de la productividad y de la mortalidad de la gallina nativa en la meseta de Sankarán Faranah, Guinea, en 1993-1994

El presente artículo trata de la productividad y de la mortalidad de la raza local de gallinas en medio aldeano (meseta de Sankarán). La productividad de esta gallina fue muy modesta, tomando en cuenta la cantidad de huevos por camada $(10,0,5 \pm 0,15)$ y el número de camadas por año $(3,8$ $\pm 0,07)$. La tasa de eclosión varió de 42 a $100 \%$. El peso medio de los huevos fue de $30,7 \pm 0,03 \mathrm{~g}$. El peso medio de los pollitos post-eclosión fue de $22,8 \pm 0,23 \mathrm{~g}$. La tasa de fertilidad fue de $87,5 \%$ y la tasa de viabilidad de los pollitos de un día fue de $89,31 \%$. La enfermedad de Newcastle y la tifosis pullorosis fueron las principales causas de mortalidad en las gallinas y pollitos (más de $60 \%$ de las pérdidas). La tasa de mortalidad bruta anual fue de $80 \%$ en las gallinas y de $73,16 \%$ en los pollitos. La tasa de mortalidad bruta estacional aumentó considerablemente durante la estación húmeda y la estación seca. El efecto de la estación sobre la mortalidad de las gallinas y de los pollitos, observado por medio de test de chi $^{2}$, fue altamente significativo $(p<0,005)$. Los coeficientes de correlación y la determinación entre estas dos variables no fueron significativos ( $p>0,05)$, siendo respectivamente iguales a $-0,639$ y 0,4081 . El intervalo de confianza del coeficiente de correlación fue de $-0,639 \pm 1,7384$. El efecto estacional sobre la eclosión no fue significativo ( $p>0,05$ ). Los efectos de las estaciones del año, la enfermedad y su interacción fueron altamente significativos $(p<0,005)$ con relación a la tasa de mortalidad de los pollitos. La relación de sexos gallina/gallo fue de $38 \%$. Los criadores se quedaron con $28 \%$ de los animales para el reemplazo del hato.

Palabras clave: Pollo - Pollito - Productividad - Crianza extensiva - Mortalidad - Enfermedad de N ewcastle - Pullorosis Método estadístico - Variación estacional - Avicultura Guinea. 